\title{
Effect of Super-Brain Yoga on the Concentrating Ability of Students
}

\author{
G. Aniruddha ${ }^{1}$, A. Santakumari ${ }^{2}$, C. Saroja ${ }^{3}$ \\ ${ }^{1}$ Postgraduate Student, ${ }^{2}$ Professor \& HOD, ${ }^{3}$ Associate Professor, \\ Upgraded Department of Physiology, Osmania Medical College
}

\begin{abstract}
Introduction: Frontal and parietal association areas form the neural basis of concentration as evidenced by cellular studies of posterior parietal cortex in monkeys. Cerebral asymmetry, an evolutionary consequence, enhances brain output by synchronizing both hemispheres for maximal brain output facilitated by Super-brain yoga - a simple,effective technique to energize and recharge the brain, assessed with $\mathrm{d} 2$ test measuring attention, scanning and mental flexibility.
\end{abstract}

Aim: To assess the effect of Super-brain yoga on the concentrating ability of students by $\mathrm{d} 2$ test.

Materials and Method: 184 school children aged 11-13 yrs, physically fit \& unaware of Super-brain yoga formed the study population.

Methodology: (1) d2 test consisting of: (i) TN: total number of characters (ii) E1: number of characters omitted (iii) E2: the number of errors (iv) $\mathbf{C P}$ : concentration performance $\mathbf{C P}=(\mathbf{T N}-\mathbf{E 2})$ was performed before and after Super-brain yoga to assess students concentrating ability. (2) Super brain yoga/yogic squats performed for 1 month.

Results: 'p' value $<0.05$ statistical significance with paired ' $t$ ' test.

Conclusion: Practicing Super-brain yoga for 1 month increased the concentrating ability of students.

Keywords: Cerebral asymmetry, d2 test, Super-brain yoga.

\section{Introduction}

Concentration is focusing the mind on a specific issue at a given moment for learning. Frontal and parietal association areas form the neural basis of concentration as evidenced by cellular studies of posterior parietal cortex in monkeys ${ }^{4}$. Super-brain yoga by Master Chao Kok Sui with crossed hands, squatting up and down is thought to increase concentrating ability.

Cerebral asymmetry of human brain is the consequence of evolution. Complementary areas of

\section{Corresponding Author:}

\section{Dr. G. Aniruddha}

Osmania Medical College

e-mail: ani3gopireddy@gmail.com both hemispheres synchronize for maximal brain output and can be achieved with Super-brain yoga- simple, effective technique to energize and recharge the brain. Human brain has 100 billion brain cells which are technically called as "Neurons" \& supporting cells as "Neuroglia". A complex system of neurons, Corpus callosum connects the brain's Right hemisphere/ Representational hemisphere/Non-dominanthemisphere and Left hemisphere/Categorical hemisphere/Dominant hemisphere ${ }^{10}$.

One needs to exercise \& nurture the brain just like physical exercise for the body to make the most of this miraculous tool ${ }^{9}$.The output of brain can be enhanced by creating $\&$ strengthening the synapse,junction between two neurons, through a physiological mechanism of "Synaptic plasticity". The brain can plasticize to maximal extent during childhood. 
$\mathrm{d} 2$ test of attention is EXECUTIVE FUNCTION TEST,categorized under neuropsychological test. The effect is assessed by $\mathrm{d} 2$ test measuring attention, scanning and mental flexibility. The current study focuses on the effectiveness of Super-brain yoga on the concentrating ability of students using $\mathrm{d} 2$ test as a key ${ }^{6}$.

\section{Materials and Method}

A pre test and post test research design was carried out at Zilla Parishat High School, Hayathnagar, Hyderabad, Telangana. This study was granted approval by the Ethics Committee of Osmania Medical College. In this study, 184 male students of $7^{\text {th }} \& 8^{\text {th }}$ classes were recruited and assigned Super-brain yoga. They were all explained about the study and motivated. Written consent was obtained from the Principal of the school. The students who were physically \& unaware of Superbrain yoga and age of 11 to 13 years were included in the study, while students who were practicing Super-brain yoga and suffering with any musculo-skeletal conditions like polio, muscular dystrophies, etc,.were excluded from the study.

d2 test consisting of: (i) TN:total number of characters (ii) E1: number of characters omitted (iii) E2:the number of errors (iv) CP:concentration performance $\mathbf{C P}=(\mathbf{T N}-\mathbf{E 2})$ was performed before and after Super-brain yoga to assess students concentrating ability. The test items consist of the letters $d$ and $P$ with one to four dashes, arranged either individually or in pairs above and below the letter. The subject must scan across each line to identify and cross out each $d$ with two dashes. The characters of letter $d$ having 2 dashes above, 2 dashes below and 1 dash above and below (correct hits) are called "relevant items." All other combinations of letters and lines are considered "irrelevant", they should not be crossed out. The one-page $\mathrm{d} 2$ test form consists of 14 lines, each with 47 characters, for a total of 658 items. The subject is allowed 20 seconds per line.

The Superbrain yoga described by Master Chao kok Sui was practiced by students at the rate of 14 Yogic squats per day ${ }^{2}$ for one month in specific hand position :i) Standing with feet slightly apart, greater than shoulder width and toes pointing forward,facing towards east. ii) Hands are crossed over each other, first left hand is crossed over upper body to take hold of right earlobe with thumb and fore finger, with thumb in front. Then right hand is crossed over left ${ }^{5}$. iii) While squatting down inhale through nose, hold the breath and exhale while coming back to standing position.

Statistical Analysis: Mean \& standard deviation for all $\mathrm{d} 2$ test parameters were derived with paired ' $\mathrm{t}$ ' test ' $p$ ' value $<0.05$ was considered statistically significant and $<0.001$ as statistically highly significant.

\section{Results}

' $p$ ' value from paired ' $t$ ' test is highly significant for all the variables of $\mathrm{d} 2$ test for selective attention.

Table: Comparison of $\mathrm{d} 2$ test parameters, before and after super-brain yoga.

\begin{tabular}{|c|c|c|c|c|}
\hline \multirow{2}{*}{ Parameters } & \multicolumn{2}{|c|}{ Mean \pm SD } & \multirow{2}{*}{ Paired t test } & \multirow{2}{*}{$p$-Value } \\
\cline { 2 - 3 } & Before & After & & \\
\hline TN & $172.30 \pm 80.969$ & $203.91 \pm 45.423$ & 7.801 & $0.001 \mathrm{HS}$ \\
\hline E1 & $159.05 \pm 64.768$ & $119.77 \pm 40.755$ & 13.771 & $0.001 \mathrm{HS}$ \\
\hline E2 & $38.29 \pm 35.433$ & $25.68 \pm 12.565$ & 6.262 & $\mathbf{0 . 0 0 1} \mathrm{HS}$ \\
\hline CP (TN-E2) & $132.23 \pm 63.748$ & $178.23 \pm 40.755$ & 13.914 & $\mathbf{0 . 0 0 1} \mathrm{HS}$ \\
\hline
\end{tabular}

$\mathrm{HS}=$ Highly significant

TN: Total attempted E1: Number of letters omitted

E2: Errors CP (TN-E2) : Concentration performance 


\section{Discussion}

The present study reveals a significant improvement in concentration \& selective attention of students after doing Super-brain yoga with 'p' value of 0.001 significance for all $\mathrm{d} 2$ test parameters. There is significance for all $\mathrm{d} 2$ test parameters $(\mathrm{p}=0.001)$. This is in agreement with "A comparative study of the effects of Super-brain Yoga \& aerobic exercise on cognitive functions" the study done by Joseph Irvin Thomas, Venkatesh $\mathrm{D}^{2}$ and study done by Srikanth N Jois, Lancy D' Souza, R Moulya on beneficial effects of Super-brain yoga on short term memory \& selective attention of students ${ }^{7}$.

In the current study all measures of $\mathrm{d} 2$ test of selective attention with ' $p$ ' value of 0.001 statistical significance correlates with "Effect of repetitive yogic squats with specific hand position (Thoppukarnam) on selective attention ${ }^{7} \&$ psychological states" by Angelica Chandrasekaran et al.

Brickenkamp and Zillmer $(1998)^{8}$ demonstrated that the $\mathrm{d} 2$ test correlates with Symbol Digit Modalities Test (Smith, 1973), Stroop Color Word Test (Stroop, 1935), and Trail Making Test Parts A and B (Reitan \& Wolfson, 1985), all of which are measures of attention, scanning and mental flexibility. The construct validity and reliability of the test has been investigated with factor analysis and against criterion measures.

To demonstrate the validity of the $\mathrm{d} 2$ test, Bates and Lemay (2004) administered $\mathrm{d} 2$ test with several other neuropsychological measures to a relatively large participant sample. The tests with measures of attention, processing speed, abstract reasoning, verbal ability, visual spatial ability and working memory were employed in their study. Results were internally consistent as measured by Cronbach's alpha.

Attentional control is the ability to direct attention to only those stimuli that are relevant to our current goals, minimizing the influences that deviate to capture attention(Corbetta and Shulman, 2002). Weber et al. (2009)proposed theory of flow which suggests widespread synchronization between neural attention networks which are the basis for the complete absorption during flow state, which is based on tripartite theory of attention proposed by Posner et al.'s (1987); involving executive, alerting and orienting networks. The alerting network is responsible for initiating and maintaining attentiveness, while the orienting network directs attention to a stimulus,both the networks are modulated by executive network. Optimal synchronization of alerting and orienting networks with executive network enhances attention to be highly goal-directed (Petersen and Posner, 2012).This organization is achieved through synchronized firing rates of neurons within attentional networks.

The importance of higher attentional processes during the flow experience is supported by neuroimaging research. Ulrich et al. (2016) have identified activation of multiple brain are asassociated with the multiple demand (MD) network during flow in an arithmetic task. Prefrontal and parietal cortex areas, including inferior frontal sulcus, anterior insula, presupplementary motor area and in and around intraparietal sulcus form the MD network. Corbetta and Shulman(2002) identified areas of parietal and frontal cortex which form frontoparietal dorsal stream ${ }^{11}$.MD system serves to coordinate a series of multistep behaviors, guide selective focus to taskrelevant information and provide cognitive control.

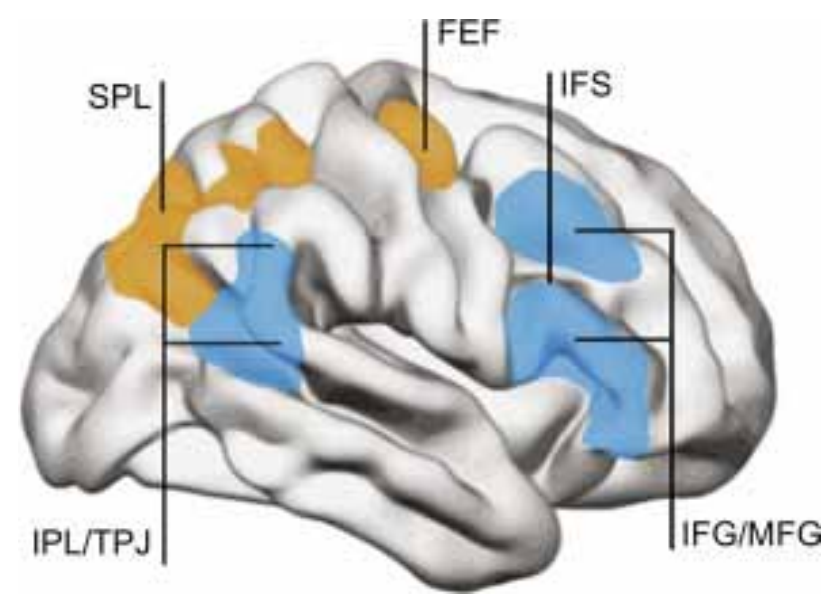

Figure: Dorsal and Ventral networks of attention

Projection from the temporoparietal junction (TPJ) toward inferior frontal gyrus (IFG) and middle frontal gyrus (MFG) form ventral network (blue), responsible for reorienting attention to salient stimuli. Projection from the superior parietal lobe (SPL) toward the frontal eye fields (FEF) form dorsal network (orange), responsible for voluntary allocation of attention. The MD system includes overlapping frontoparietal areas, from the SPL to the premotor cortex and inferior frontal sulcus (IFS).

Neurotransmitter activity of dopamine affectattentional processes. Dopamine pathways are primarily associated with reward networks and also modulate attentional focusing (Nieoullon, 2002), error 
monitoring (Holroyd and Coles, 2002; Ridderinkhof et al., 2004) and response inhibition (Chambers et al., 2009; Congdon et al., 2008).Increased availability of dopamine D2 receptors in the striatum are functionally related to selective attention (Nieoullon, 2002). Dopamine actionmodulatesresponse inhibition and impulse control for attentional control (Miyake et al., 2000).

The neural basis of concentration lies between the frontal and parietal association areas. When Super-brain yoga is practiced it is thought that, strengthening of synapses occur by physiological mechanism of synaptic plasticity due to increased synchronized firing rates of neurons within attentional networks and Corpus callosum as well which is responsible for synchronization of the two hemispheres giving out maximal output of the brain.

Master Choa kok Sui explains that the Super-brain yoga - YOGIC SQUATS when done correctly moves energy trapped in the basic chakras through the physical body's other major energy centers and finally up into the crown chakra that controls the pineal gland and overall brain health ${ }^{1,2}$. "Once the energy gets up to the forehead and crown chakras, it is transformed into subtle energy, which is utilized by the brain for its proper functioning," writes Master Choa kok Sui.

Jeremy Vanhelst et al., conducted a study on "Physical activity is associated with attention capacity in adolesecents ${ }^{13}$ " concluded that promoting Moderateto-vigorous physical activity may be have a beneficial effect on attention capacity in adolescents. In the present study students doing regular exercise were not excluded as exercise was part of their regular school schedule. Further a study comparing both Super-brain yoga and moderate exercise is to be done.

\section{Conclusion}

Instituting Super-brain yoga for one month are effective in enhancing concentrating ability of students as evidenced by:

1. Significant increase in:

TN: Total number of characters attempted and

$\mathbf{C P}=($ TN-E2): Concentration performance

2. Significant decrease in:

E1: Number of letters omitted and

E2: Errors committed
Acknowledgments: We acknowledge the support of faculty \& post graduate students of Upgraded department of physiology, Osmania Medical College, Hyderabad and the study population in conducting the study.

Ethical Clearance: Taken from Osmania Medical College Institutional ethics committee

\section{Source of Funding: Self}

\section{Conflict of Interest: Nil}

\section{References}

1. Verma S, Kumar K. Evidence based study on super brain yoga and its application on alpha EEG in adolescence. Int J Sci Consciousness. 2016;2:40-6.

2. Thomas JI, Venkatesh D.A comparative study of the effects of superbrain yoga and aerobic exercise on cognitive functions. National Journal of Physiology, Pharmacy and Pharmacology. 2017;7(9):895.

3. Sethi JK, Nagendra HR, Ganpat TS. Yoga improves attention and self-esteem in underprivileged girl student. Journal of education and health promotion. 2013;2.

4. Eric r. Kandel. James h. Schwartz, thomas m. Jessell, steven a. Siegelbaum, a. J. Hudspeth. Principles of neural science - fifth edition.

5. Chandrasekeran A, Rajesh SK, Srinivasan TM. Effect of repetitive yogic squats with specific hand position (Thoppukaranam) on selective attention and psychological states. International journal of yoga. 2014 Jan;7(1):76.

6. Lezak MD, Howieson DB, Loring DW, Fischer JS. Neuropsychological assessment. Oxford University Press, USA; 2004.

7. Cowan N. What are the differences between longterm, short-term, and working memory? Prog Brain Res. 2008;169:323-38.

8. Bates ME, Lemay EP. The d2 Test of attention: construct validity and extensions in scoring techniques. Journal of the International Neuropsychological Society. 2004 May;10(3):392400.

9. North V, Israel R. Mind Chi: Re-wire Your Brain in 8 Minutes a Day--Strategies for Success in Business and Life. John Wiley \& Sons; 2010 Jan 5.

10. Kim E. Barrett, phd; Scott Boitano, phd; Susan M. 
Barman, phd; Heddwen L. Brooks, phd. Ganong's Review of Medical Physiology TWENTY-FIFTH EDITION;

11. Wilson M, Walsh V, Parkin B. Sport and the Brain: The Science of Preparing, Enduring and Winning. Academic Press; 2017 Oct 11.

12. Sui CK. Superbrain yoga. Institute for Inner Studies
Publishing; 2005.

13. Jeremy Vanhelst et al. Physical activity is associated with attention capacity in adolesecents. The journal of pediatrics, publishing 2015.

14. John E. Hall. Guyton and Hall Textbook of Medical physiology $13^{\text {th }}$ edition. 